\title{
Open source software selection using an analytical hierarchy process (AHP)
}

\author{
Yusmadi Yah Jusoh ${ }^{1, *}$, Khadijah Chamili ${ }^{2}$, Noraini Che Pa ${ }^{1}, J$ Jamaiah H. Yahaya ${ }^{3}$ \\ ${ }^{1}$ Dept. of Software Engineering and Information Systems, Faculty of Computer Science and Information Technology, Universiti Putra \\ Malaysia (UPM), Serdang, Selangor, Malaysia \\ ${ }^{2}$ Centre of Information Technology, USIM, Nilai, Negeri Sembilan, Malaysia \\ ${ }^{3}$ School of Computer Science, Faculty of Information Science and Technology, National University of Malaysia (UKM), Bangi, Selangor, \\ Malaysia
}

\section{Email address:}

yusmadi@upm.edu.my (Y. Y. Jusoh), khadijah@usim.edu.my (K. Chamili), norainip@upm.edu.my (N. C. Pa), jhy@ftsm.ukm.my (J. H. Yahaya)

\section{To cite this article:}

Yusmadi Yah Jusoh, Khadijah Chamili, Noraini Che Pa, Jamaiah H. Yahaya. Open Source Software Selection Using an Analytical Hierarchy Process (AHP). American Journal of Software Engineering and Applications. Vol. 3, No. 6, 2014, pp. 83-89.

doi: 10.11648/j.ajsea.20140306.13

\begin{abstract}
There are many potential and good open source software (OSS) products available on the market with a free license. However, with various choices, the adoption rate is still low among potential users because there is not an agreed acceptable set of criteria to evaluate and select various OSS. The criteria of selection may differ between the stakeholders within the organisations. There is a tendency that the user may have a biased perception of an OSS's characteristics or capabilities for solving problems when selecting OSS products. Other restrictions are caused by inadequate documentation and user manuals, and immature products. Therefore, the users need to consider how to improve their decision making when selecting the most suitable OSS products. In this paper, the background research on the proposed OSS adoption and criteria of selection are discussed and explored. Then the research methodology, processes and implementation of the My Open Source Software Toolkit (MyOSST) v1.0 are covered. The analytical hierarchy process (AHP) was applied on the selection process and for the purpose of assisting the potential user to decide on the OSS products based on their preferred selection criteria. MyOSST v1.0 was tested and validated by IT professionals in one of the Malaysian universities. The results show that the tool is capable of assisting the decision making process for selecting an appropriate OSS product.
\end{abstract}

Keywords: Open Source Software Selection, Selection Criteria, Analytical Hierarchy Process

\section{Introduction}

The adoption of open source software (OSS) is still growing all over the world, including within businesses, non-profits and public sector agencies because of the financial benefits. The criteria of selection may differ between the stakeholders within the organizations. Therefore, there is a tendency for the user to have a biased perception of an OSS's characteristics or capabilities for solving problems when selecting OSS products.

The OSS adoption rate in Malaysian public sector agencies was still low up until end of 2010 [1]. Therefore, the next step is to apply the suggested selection criteria to understand what is the best dimension to contribute to Malaysian public sector agencies in order to encourage the adoption and implementation of OSS products in their agencies [2] [3]. In other words, the proposed selection criteria may help to identify the characteristics to be considered by the users when adopting the OSS product. Hence, conducting a survey may give support to the identification of the main characteristics for the proposed OSS adoption model.

In our previous study, we identified all the possible OSS characteristics which referred to the ISO standard for comparison, better understanding and future enhancement purposes. We also proposed an additional quality characteristic which is the internal constraint to be included when agencies make decisions concerning OSS adoption. With these hierarchical characteristics of OSS, the selection criteria might benefit users by building confidence in OSS product adoption in the future [4].

Recently, there have been many potential and good OSS 
products on the market with a free license, but yet they are still not popular among Malaysian public sector agencies. What are the constraints or limitations on adopting these good OSS products? What drives them to rely on proprietary software which costs them hundreds of thousands of dollars? To understand this scenario, a survey was conducted among technical and IT managerial personnel at selected public sector agencies. Identifying the preferred selection criteria will help to build confidence among users and a better understanding of their perception of OSS.

The remainder of the paper is organized as follows. In Section 2, the proposed OSS selection dimensions and criteria are presented, and section 3 discusses the methodology which includes the AHP, the selection processes and tool development. Sections 4 explain the MyOSST v1.0 implementation, a toolkit to assist the OSS product selection process. The results and discussion are presented in Section 5. Finally, in Section 6 the insights of the study are summarised and directions are given for future research.

The next section will explain the OSS selection dimensions. The selections are based on four dimensions: system quality, information quality, service quality, and another dimension which includes the potential internal constraints.

\section{The OSS Selection Dimensions}

The first step of our study was to explore the existing research on the selection criteria for the OSS product. Many researchers have suggested models and frameworks for their preferred list of important criteria for OSS selection. The detail of related studies was reported in a previous publication [4] [5].

It is important to note that in generating the selection criteria in this study, we referred mainly to four main sources: (i) the D\&M IS Success Model [6] (ii) related work on the OSS quality model [7] [8] [9] [10] [11] and (iii) general standards for software quality, such as ISO 25010 [12] and (iv) the Malaysian Public Sector Open Source Software Initiative: OSS Implementation Guidelines [3].

The suggested selection criteria consist of four (4) dimensions: system quality, information quality, service quality and one other dimension. The additional dimension is named as other and its criteria and characteristics can be added by the organization from their own evaluation criteria such as internal technical competencies and knowledge. The proposed dimensions, criteria and characteristics for selecting the OSS product are as shown in Table 1 .

Table 1. The dimensions, criteria and characteristics.

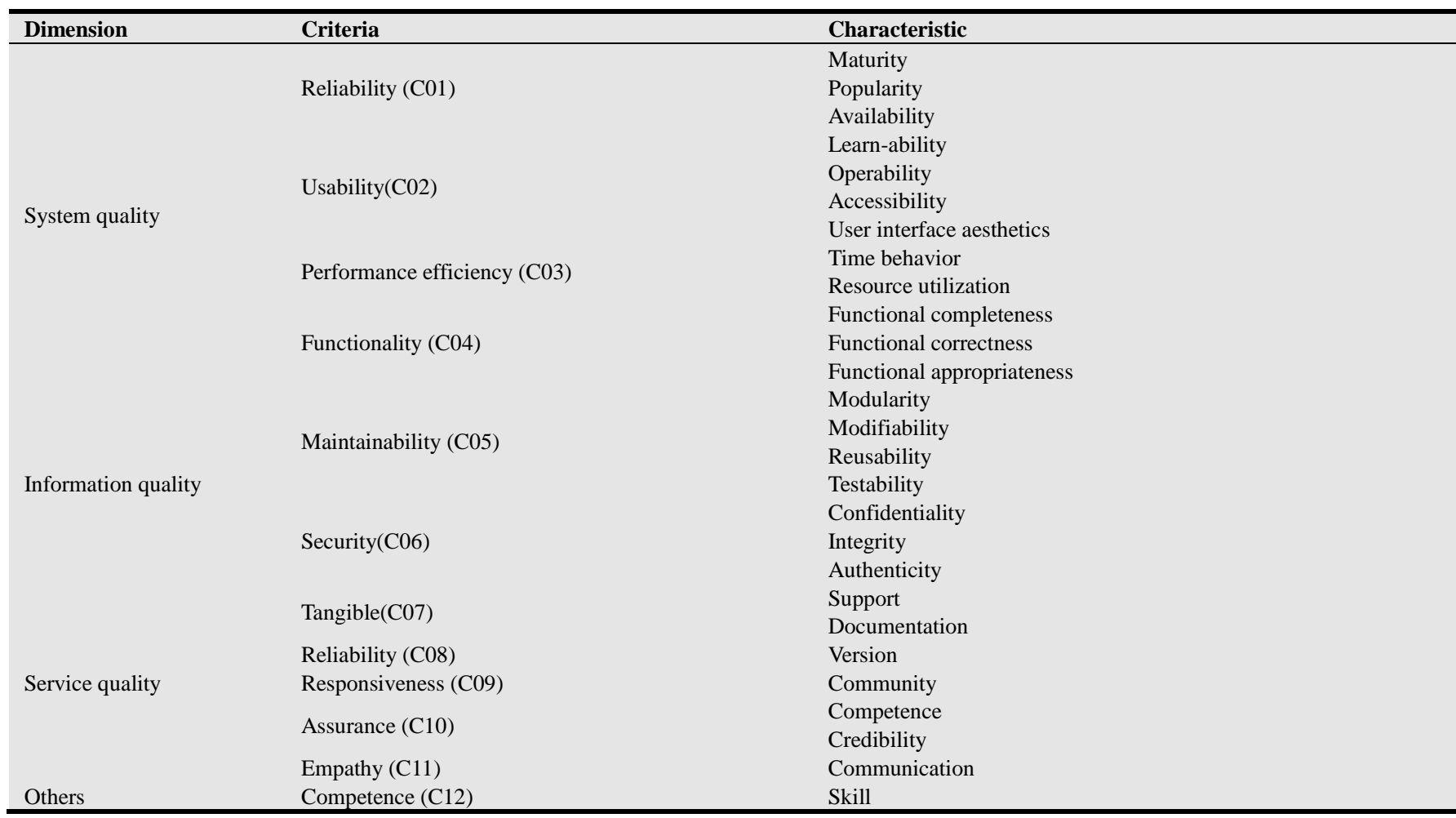

The dimensions of the OSS product selection are organized in a hierarchy of two levels which are the criteria and characteristics. The dimensions include 12 criteria for selection. Each criterion has one or more characteristics. The characteristics also can be added by the organization to comply with their organizational requirements for the OSS products.
Both the criteria and the characteristics are considered to contribute in some way or other to each of the dimensions that they belong to. We also suggest the measurement for each of the sub-characteristics to ensure that these quality characteristics are ranked accordingly [5]. 


\section{Methodology}

In this section, we will describe the methodology of the proposed OSS selection dimensions which were included in the toolkit that was developed, and elaborate the AHP technique applied for the decision making for selecting the OSS products and the development environment of the toolkit, MyOSST v1.0.

\subsection{Analytical Hierarchy Process (AHP)}

The AHP was introduced by Thomas L. Saaty in the 1970s. It is a systematic decision making method which includes both qualitative and quantitative techniques. It has been widely used for a long time in many fields. The AHP is also a structured technique for organizing and analysing complex decisions based on mathematics and psychology [13] [14].

Rather than prescribing a "correct" decision, the AHP helps decision makers find one that best suits their goal and their understanding of the problem. It provides a comprehensive and rational framework for structuring a decision problem, for representing and quantifying its elements, for relating those elements to overall goals, and for evaluating alternative solutions.

The main advantage of the AHP is its ability to rank choices in the order of their effectiveness in meeting conflicting objectives. It is quite hard - but not impossible - to 'fiddle' the judgments to get some predetermined result as well as to detect inconsistent judgments.

The AHP can also be used by individuals working on uncomplicated decisions; it is most useful where teams of people are working on complex problems, especially those with high stakes, involving human perceptions and judgments, whose resolutions have long-term repercussions. It has unique advantages when important elements of the decision are difficult to quantify or compare, or where communication among team members is impeded by their different specializations, terminologies, or perspectives.

Users of the AHP first decompose their decision problem into a hierarchy of more easily comprehended sub-problems, each of which can be analysed independently. The elements of the hierarchy can relate to any aspect of the decision problem-tangible or intangible, carefully measured or roughly estimated, well- or poorly-understood-anything at all that applies to the decision at hand.

Once the hierarchy is built, the decision makers systematically evaluate its various elements by comparing them to one another two at a time, with respect to their impact on an element above them in the hierarchy. In making the comparisons (the pair-wise comparison), the decision makers can use concrete data about the elements, but they typically use their judgments about the elements' relative meaning and importance. Each of these judgments is assigned a number on a scale as shown in Table 2.

These pair-wise comparisons are carried out for all elements to be considered, until the matrix is completed. It is the essence of the AHP that human judgments, and not just the underlying information, can be used in performing the evaluations. The AHP converts these evaluations to numerical values that can be processed and compared over the entire range of the problem. A numerical weight or priority is derived for each element of the hierarchy, allowing diverse and often incommensurable elements to be compared to one another in a rational and consistent way. This capability distinguishes the AHP from other decision making techniques.

In the final step of the process, numerical priorities are calculated for each of the decision alternatives. These numbers represent the alternative's relative ability to achieve the decision goal, so they allow a straightforward consideration of the various courses of action.

Table 2. AHP Scale.

\begin{tabular}{lll}
\hline Intensity of Importance & Definitions & Explanations \\
\hline 1 & Equal & Two factors contribute equally to the objective \\
3 & Moderate & Experience and judgments slightly favors one over the other. \\
5 & Strong & Experience and judgments strongly favor one over the other. \\
7 & Very Strong & $\begin{array}{l}\text { Experience and judgments very strongly favor one over the other. Its importance is } \\
\text { demonstrated in practice. }\end{array}$ \\
9 & Extreme & The evidence favoring one over the other is of the highest possible validity. \\
$2,4,6,8$ & When compromise is needed \\
\hline
\end{tabular}

\subsection{The Selection Process}

The processes for selecting the OSS products are proposed to identify the best alternatives or preferences by implementing the AHP. There are four processes involved as depicted in Figure1.

The first process of the selection is to establish the AHP structure by identifying the selected selection criteria. In this step the hierarchy of criteria and the alternatives are generated. Therefore, there are three important components which need to be identified: the goal/project, the criteria of the products, and the list of alternatives/products.

In the next process, human or user judgment is needed.
The user needs to determine the relative importance of each criterion, which means ranking the criteria based on the priorities for each of the criteria using the matrix.

Next, the judgment exercise involves applying the pair-wise comparison for each of the criteria using the proposed AHP scale in Table 2. For example C02-System Usability is 3 times as importance as C01-System Reliability.

After completing the judgment matrix, the last process is to multiply the alternative weight by the criteria weight to obtain the best of all the alternatives. Finally, the result of the final ranking was computed and presented for decision making to the selection committee or users. 


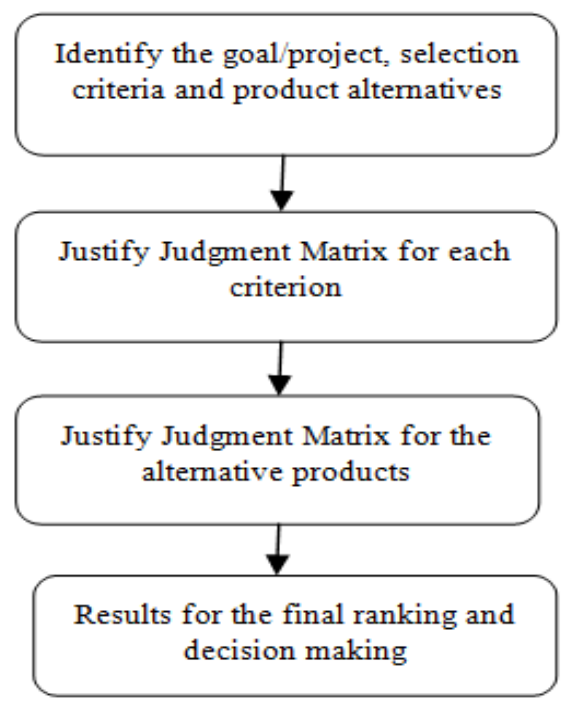

Figure 1. The Selection Process.

\subsection{The Development}

In developing the OSS selection criteria toolkit, a few requirements have been identified. First, the system should be a web based application to allow users to access it via the internet. The development uses the OSS environment - that is MySQL 4.1.14, PHP 5.0.5, and Apache HTTP Server.

MySQL is the world's most used open source relational database management system (RDBMS), which runs as a server providing multi-user access to a number of databases. MySQL ships with no GUI tools for administering MySQL databases or managing the data contained within the databases. Users may use the included command line or download MySQL front-ends from various parties that have developed desktop software and web applications to manage MySQL databases, build database structures, and work with data records. The data in MySQL is stored in database objects called tables. A table is a collection of related data entries and it consists of columns and rows. Databases are useful when storing information categorically.

PHP is a general-purpose server-side scripting language originally designed for Web development to produce dynamic Web pages. It is one of the first server-side scripting languages to be embedded into an HTML source document rather than calling an external file to process data. The code is interpreted by a Web server with a PHP processor module which generates the resulting Web page. It has also evolved to include a command-line interface capability and can be used in standalone graphical applications. PHP can be deployed on most Web servers and also as a standalone shell on almost every operating system and platform free of charge.

The Apache HTTP Server, commonly referred to as Apache, is web server software notable for playing a key role in the initial growth of the World Wide Web. In 2009 it became the first web server software to surpass the 100 million website milestone. Apache was the first viable alternative to the Netscape Communications Corporation web server (currently named Oracle iPlanet Web Server), and since has evolved to dominate other web servers in terms of functionality and performance. Typically, Apache is run on a Unix-like operating system.

\section{The Implementation}

MyOSST has been developed to implement all validated OSS selection criteria in order to assist potential users while making decisions on OSS products in their agencies. Below is a list of the scope and features of the system:

- web based application which allows users access via the internet.

- adopting the OSS selection dimension-criteria which are system-reliability (C01), system-usability (C02), system-performance efficiency (C03), system-functionality (C04), information-maintainability (C05), information-security (C06), service-tangible (C07), service-reliability (C08), service-responsiveness (C09), service-assurance (C10), service-empathy (C11) and other-competence (C12).

- allows single input (one person) for each project based.

Small group discussions are encouraged to determine the criteria weightage. MyOSST v1.0 was developed using the suggested methodology for AHP as depicted in Figure 1. The illustrations given are used only as an example on the implementation of the particular system.

\subsection{Identify the Goal, Selection Criteria and Product Alternatives}

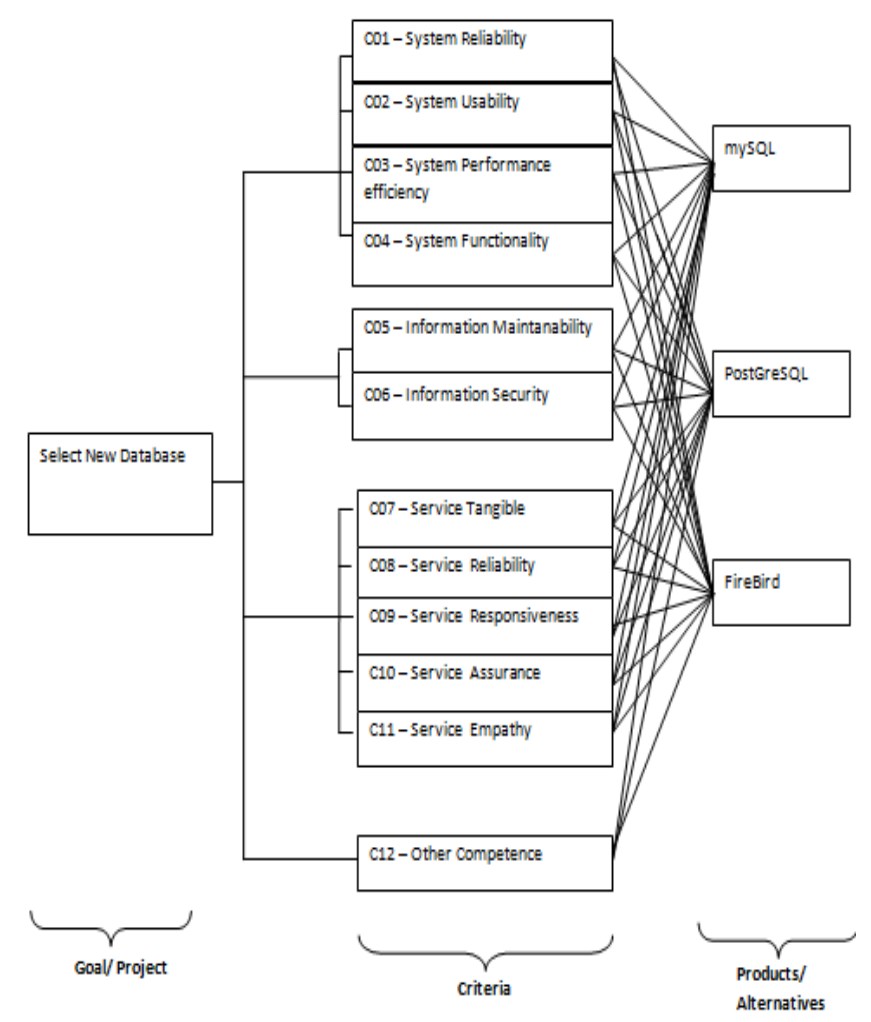

Figure 2. AHP Structure for Select New Database.

In MyOSST v1.0, the criteria have been defined which are applied from Table 1. Further to this section, we demonstrate 
how AHP is applied in MyOSST v1.0 as Select New Database, which is shown as the goal or project presented in this paper. The complete AHP structure for Select New Database is as depicted in Figure 2.

\subsection{Judgment Matrix for Each Criterion}

Using pair-wise comparison, the relative importance of one criterion over another can be expressed as depicted in Figure 3.

\begin{tabular}{|c|c|c|c|c|c|c|c|c|c|c|c|c|}
\hline \multicolumn{13}{|c|}{ Matrix } \\
\hline & $\mathrm{C01}$ & $\mathrm{CO2}$ & $\mathrm{C} 03$ & C04 & $\cos$ & C06 & C07 & C08 & $\mathrm{CO9}$ & C10 & C11 & C12 \\
\hline$C 01$ & 1 & $1 / 3$ & $1 / 7$ & $1 / 5$ & $2 / 1$ & $1 / 9$ & $1 / 5$ & $1 / 2$ & $1 / 2$ & $1 / 5$ & $1 / 2$ & $1 / 5$ \\
\hline $\mathrm{CO2}$ & 3/1 & 1 & $1 / 5$ & $1 / 2$ & $5 / 1$ & $1 / 8$ & $1 / 5$ & $5 / 1$ & $5 / 1$ & $1 / 5$ & $3 / 1$ & $1 / 3$ \\
\hline $\mathrm{CO3}$ & 7/1 & $5 / 1$ & 1 & 3/1 & $7 / 1$ & $1 / 2$ & $1 / 2$ & $7 / 1$ & 7/1 & $5 / 1$ & $7 / 1$ & $5 / 1$ \\
\hline C04 & $5 / 1$ & $2 / 1$ & $1 / 3$ & 1 & $7 / 1$ & $1 / 3$ & $1 / 3$ & $5 / 1$ & $5 / 1$ & $1 / 4$ & $5 / 1$ & $1 / 3$ \\
\hline $\cos$ & $1 / 2$ & $1 / 5$ & $1 / 7$ & $1 / 7$ & 1 & $1 / 9$ & $1 / 8$ & $1 / 2$ & $1 / 2$ & $1 / 7$ & $1 / 2$ & $1 / 5$ \\
\hline C06 & 9/1 & $8 / 1$ & $2 / 1$ & $3 / 1$ & 9/1 & 1 & 2/1 & $8 / 1$ & $8 / 1$ & $6 / 1$ & 9/1 & $3 / 1$ \\
\hline$C 07$ & $5 / 1$ & $5 / 1$ & $2 / 1$ & $3 / 1$ & $8 / 1$ & $1 / 2$ & 1 & $5 / 1$ & $7 / 1$ & $2 / 1$ & $3 / 1$ & $2 / 1$ \\
\hline C08 & 2/1 & $1 / 5$ & $1 / 7$ & $1 / 5$ & $2 / 1$ & $1 / 8$ & $1 / 5$ & 1 & $1 / 3$ & $1 / 5$ & $1 / 4$ & $1 / 7$ \\
\hline C09 & 2/1 & $1 / 5$ & $1 / 7$ & $1 / 5$ & $2 / 1$ & $1 / 8$ & $1 / 7$ & $3 / 1$ & 1 & $1 / 5$ & $1 / 2$ & $1 / 5$ \\
\hline C10 & $5 / 1$ & $5 / 1$ & $1 / 5$ & $4 / 1$ & 7/1 & $1 / 6$ & $1 / 2$ & $5 / 1$ & $5 / 1$ & 1 & $2 / 1$ & $3 / 1$ \\
\hline C11 & $2 / 1$ & $1 / 3$ & $1 / 7$ & $1 / 5$ & $2 / 1$ & $1 / 9$ & $1 / 3$ & $4 / 1$ & 2/1 & $1 / 2$ & 1 & $1 / 5$ \\
\hline C12 & $5 / 1$ & $3 / 1$ & $1 / 5$ & $3 / 1$ & $5 / 1$ & $1 / 3$ & $1 / 2$ & 7/1 & $5 / 1$ & $1 / 3$ & $5 / 1$ & 1 \\
\hline
\end{tabular}

Figure 3. The Pair-wise Matrix.

After completing the matrix for each criteria, AHP turns the matrix into a ranking of criteria. To obtain the ranking of priorities from the pair-wise matrix, an eigenvector solution is applied. Below is the step by step process to perform the eigenvector:

1) Converting the fraction value to the decimal number as shown in Figure 4.

\begin{tabular}{|c|c|c|c|c|c|c|c|c|c|c|c|c|}
\hline \multicolumn{13}{|c|}{ Matrix } \\
\hline & $\mathrm{C}_{01}$ & $\mathrm{CO2}$ & $\mathrm{CO3}$ & $\mathrm{CO4}$ & C05 & C06 & $\mathrm{CO7}$ & $\cos$ & Co9 & C10 & C11 & C12 \\
\hline $\mathrm{C} 01$ & 1.00 & 0.33 & 0.14 & 0.20 & 2.00 & 0.11 & 0.20 & 0.50 & 0.50 & 0.20 & 0.50 & 0.20 \\
\hline $\mathrm{CO} 2$ & 3.00 & 1.00 & 0.20 & 0.50 & 5.00 & 0.13 & 0.20 & 5.00 & 5.00 & 0.20 & 3.00 & 0.33 \\
\hline $\mathrm{C} 03$ & 7.00 & 5.00 & 1.00 & 3.00 & 7.00 & 0.50 & 0.50 & 7.00 & 7.00 & 0.20 & 7.00 & 5.00 \\
\hline C04 & 5.00 & 2.00 & $1 / 3$ & 1.00 & 7.00 & 0.33 & 0.33 & 5.00 & 5.00 & 0.25 & 5.00 & 0.33 \\
\hline $\cos$ & 0.50 & 0.20 & 0.14 & 0.14 & 1.00 & 0.11 & 0.13 & 0.50 & 0.50 & 0.14 & 0.50 & 0.20 \\
\hline C06 & 9.00 & 8.00 & 2.00 & 3.00 & 9.00 & 1.00 & 2.00 & 8.00 & 8.00 & 6.00 & 9.00 & 3.00 \\
\hline $\mathrm{CO}$ & 5.00 & 5.00 & 2.00 & 3.00 & 8.00 & 0.50 & 1.00 & 5.00 & 7.00 & 2.00 & 3.00 & 2.00 \\
\hline C08 & 2.00 & 0.20 & 0.14 & 0.20 & 2.00 & 0.13 & 0.20 & 1.00 & 0.33 & 0.20 & 0.25 & 0.14 \\
\hline $\cos$ & 2.00 & 0.20 & 0.14 & 0.20 & 2.00 & 0.13 & 0.14 & 3.00 & 1.00 & 0.20 & 0.50 & 0.20 \\
\hline $\mathrm{C} 10$ & 5.00 & 5.00 & 5.00 & 4.00 & 7.00 & 0.17 & 0.50 & 5.00 & 5.00 & 1.00 & 2.00 & 3.00 \\
\hline C11 & 2.00 & 0.33 & 0.14 & 0.20 & 2.00 & 0.11 & 0.33 & 4.00 & 2.00 & 0.50 & 1.00 & 0.20 \\
\hline $\mathrm{C} 12$ & 5.00 & 3.00 & 0.20 & 3.00 & 5.00 & 0.33 & 0.50 & 7.00 & 5.00 & 0.33 & 5.00 & 1.00 \\
\hline
\end{tabular}

Figure 4. Convert to Decimal Numbers.

2) Squaring the pair-wise matrix where the matrix is multiplied with itself. Each value of each column $(\mathrm{C} 1$, $\mathrm{C} 2$...C12) is multiplied with each value of each column (R1, R2 ...R12) to obtain a new value in each cell of the new matrix.
3) Sum each of the rows of the matrix

4) Sum the total rows of the matrix

5) Normalize by dividing each of the sum rows by the total rows. The value of the last step is the eigenvector of the pair-wise matrix. The computed eigenvector value gives us the relative ranking of our criteria.

\subsection{Judgment Matrix for the Alternative Products}

Once the eigenvector value is computed, users need to repeat the pair-wise comparison activities for the alternatives on each of the OSS selected criteria. In this activity, users determine the preferences for each alternative over another such as for the criteria of system reliability (C01) with the fraction numbers for the pair-wise comparison on the alternatives.

After completing the pair-wise comparison on the alternatives, the weights for each alternative are generated using the eigenvector solution. Computing the eigenvector determines the relative ranking of the alternatives under each criterion.

\subsection{The Final Ranking and Decision Making}

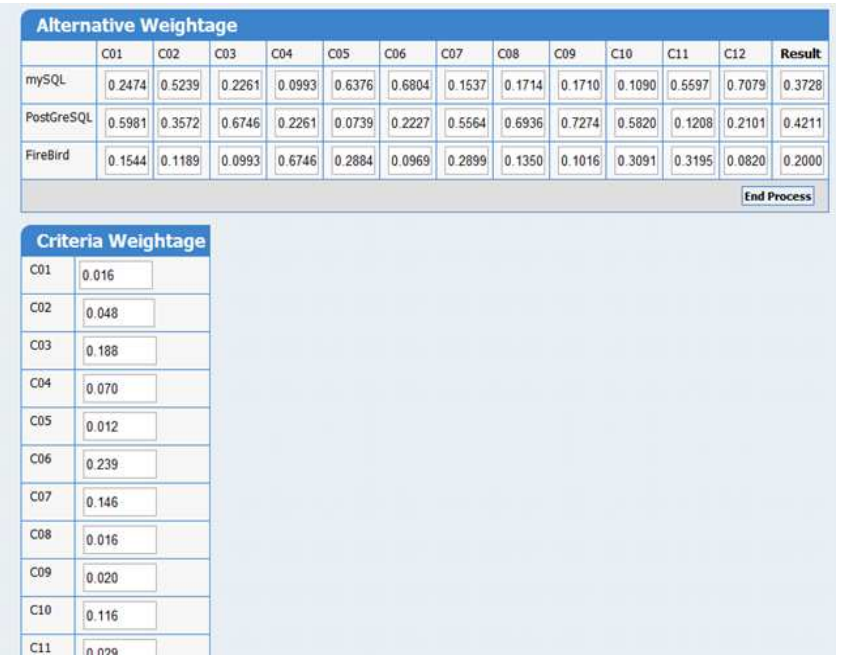

Figure 5. Alternative and Criteria Weightage

Lastly, the tool will multiply the alternative weight with the criteria weight to obtain the winner amongst all the alternatives. The result of the selection is presented as shown in Figure 5.

\section{Results and Discussion}

MyOSST v1.0 was tested in one of the Malaysian public universities. The testing exercise was conducted within a half day session at the Data Centre Unit, Information Technology Centre in June 2012. The testing session is started by explaining the objective of the testing session to a small group from the Data Centre Unit which involves the Senior IT officer, the IT officer and the assistant IT officer. Coincidently, the team is currently evaluating three business intelligent (BI) tools. They have studied product alternatives and have already 
installed, tested and previewed the selected products. However, the selected products are not $100 \%$ open source products. They are a mix between open source and proprietary products.

The products/alternatives list for Business Intelligent (BI) tools consists of OracleBI (proprietary), Qlikview, and Pentaho (OSS). After some discussion, we decided to continue the testing session, which we believed could give better results when using MyOSST v1.0 for decision making concerning choosing the precise tool.

They were given a briefing on how to use MyOSST v1.0 and how to prepare the pair-wise matrix. The user was also informed of the employment of the AHP technique as a core function of the OSS decision making tool. The users were then given half an hour to understand the criteria and prepare the pair-wise matrix manually. Finally, they used MyOSST v1.0 by themselves with guidance from the instructor.

As a result, the group found that this toolkit was very useful and really helped them in selecting a product which was based on the pair-wise comparison and selection criteria. However, they found that it was quite difficult to prepare the pair-wise comparison, which needed them to understand and technically compare the criteria. But they agreed that this technique would give a more accurate result rather than the traditional way which they needed to choose based on a one-one product features comparison which may lead to unfair judgments.

Another suggestion highlighted by them is that they need to have a detailed discussion with other team members before creating the pair-wise comparison again, and obtain a consensus result for their proposal writing. In future it is suggested that the toolkit is capable of generating a pair-wise comparison based on multiple users for group decision making.

\section{Conclusion}

This paper describes the OSS selection criteria as well as the development tools and technique used. The Analytical Hierarchy Process (AHP) is one of the best decision making techniques used all over the world. This technique was adopted in MyOSST v1.0 in order to assist in the decision making of OSS products selection. MyOSST v1.0 will hopefully assist potential OSS users on selecting any products using the OSS selection criteria.

Based on the tool implementation at one of the data centres in one of the Malaysian universities, some positive feedback and suggestions for improvements were obtained. The next step is to make some modifications and then provide further exposure in other organisations.

The study of a methodology to improve the decision making concerning OSS selection may help to identify an appropriate OSS selection solution more easily. Therefore using fuzzy theory, those dimensions, criteria and characteristics can possibly be transformed into a hierarchical structure that represents the weight of importance for the users. Group decision making should also be considered in future, since several decision makers are involved [15].

\section{Acknowledgements}

We are grateful for the Universiti Putra Malaysia's (UPM) financial support for the project.

\section{References}

[1] OSS Adoption Statistics. 2010. Malaysian Public Sector Open Source Software Programme. OSCC MAMPU. http://www.oscc.org.my/content/view/227/139/. Accessed on Jan 8, 2012.

[2] Malaysian Administrative Modernization and Management Planning Unit (MAMPU). 2011. The Malaysian Government Interoperability Framework for Open Source Software (MyGIFOSS). http://www.oscc.org.my//content/view/185/245/. Accessed on Dec 25, 2011.

[3] Malaysian Administrative Modernisation and Management Planning Unit (MAMPU). 2011. Malaysian Public Sector Open Source Software (OSS) Initiative, Open Source Software (OSS) Implementation Guidelines. http://www.oscc.org.my/content/view/186/244/. Accessed on Dec 26, 2011.

[4] Khadijah Chamili, Yusmadi Yah Jusoh, Jamaiah H. Yahaya, Noraini Che Pa. 2012. Selection Criteria for Open Source Software Adoption in Malaysia. Asian Transaction on Basic and Applied Sciences. 2 (2). ISSN: 2221-4291. On-line Journal.

[5] Khadijah Chamili, Yusmadi Yah Jusoh, Jamaiah H. Yahaya, Noraini Che Pa. 2012. The Selection Criteria for Open Source Software Adoption in Malaysia. International Journal of Advancement in Computing Technology (IJACT). In press.

[6] W. H. Delone, E. R. McLean. 2003. The DeLone and McLean Model of Information Systems Success: A Ten Year Update. Journal of Management Information Systems. Springer. 19 (4): 9-30.

[7] N. Ahmad. 2011. A Systematic Approach to Evaluating Open Source Software. International Journal of Strategic Information Technology and Applications. 2 (1): 48-67. Doi: 10.4018/jsita.2011010104.

[8] J. Confino, P. Laplante. 2010. An Open Source Software Evaluation Model. International Journal of Strategic Information Technology and Applications. IGI Global. 1 (1): 60-77.

[9] E. Glynn, B. Fitzgerald, C. Exton. 2005. Commercial adoption of open source software: An empirical study. International symposium on Empirical Engineering. pp. 225-234.

[10] D. Spinellis, V. Giannikas. 2012. Organizational adoption of open source software. Journal of Systems and Software. 85 (3): 666-682.

[11] R. Uzma, M. J. Tretter. 2012. Defining and evaluating a measure of open source project survivability. IEEE Transactions on Software Engineering. 38 (1).

[12] ISO/IEC 25010:2011 Systems and software engineeringsystems and software quality requirement and evaluation (SquaRE) - system and software quality models.

[13] T. L. Saaty. 1980. The Analytical Hierarchy Process. New York: McGraw Hill. 
[14] T. L. Saaty. 1986. Axiomatic Foundation of the Analytical Hierarchy Process. Management Science. 32(7): 841-855.
[15] M. A. Azadeh, S. N. Shirkouhi. 2009. Evaluating simulation software using fuzzy analytical hierarchy process. In Proceedings of SpringSim, 2009. 